\title{
Peripartum cardiomyopathy and dilated cardiomyopathy: different at heart
}

\author{
IIse A. E. Bollen, Elza D. Van Deel, Diederik W. D. Kuster and Jolanda Van Der Velden* \\ Department of Physiology, Institute for Cardiovascular Research (ICaR-VU), VU University Medical Center, Amsterdam, Netherlands
}

\section{Edited by:}

Julien Ochala, KIng's College

London, UK

\section{Reviewed by:}

Martina Krüger, Heinrich Heine University Düsseldorf, Germany

Ranganath Mamidi, Case Western

Reserve University, USA

\section{*Correspondence:}

Jolanda Van Der Velden,

Department of Physiology, Institute

for Cardiovascular Research, VU

University Medical Center, Van der

Boechorststraat 7, 1081 BT

Amsterdam, Netherlands

e-mail: j.vandervelden@vumc.nl
Peripartum cardiomyopathy (PPCM) is a severe cardiac disease occurring in the last month of pregnancy or in the first 5 months after delivery and shows many similar clinical characteristics as dilated cardiomyopathy (DCM) such as ventricle dilation and systolic dysfunction. While PPCM was believed to be DCM triggered by pregnancy, more and more studies show important differences between these diseases. While it is likely they share part of their pathogenesis such as increased oxidative stress and an impaired microvasculature, discrepancies seen in disease progression and outcome indicate there must be differences in pathogenesis as well. In this review, we compared studies in DCM and PPCM to search for overlapping and deviating disease etiology, pathogenesis and outcome in order to understand why these cardiomyopathies share similar clinical features but have different underlying pathologies.

Keywords: dilated cardiomyopathy, peripartum cardiomyopathy, oxidative stress, prolactin, microvasculature, heart failure, titin

\section{INTRODUCTION}

Peripartum cardiomyopathy (PPCM) is a cardiac disease that can have many different clinical presentations but shares similar clinical symptoms with dilated cardiomyopathy (DCM) such as ventricle dilation, impaired systolic function and arrhythmias (Pearson et al., 2000; Elliott et al., 2008). Therefore, PPCM is often seen as DCM initiated during pregnancy. However, there are some important differences between the two cardiomyopathies which argue for separation of the two disease states. This review aims to compare studies performed in PPCM and DCM patients and animal models in order to better understand why these cardiomyopathies share similar clinical features but have different underlying pathomechanisms.

\section{CLINICAL PRESENTATIONS}

Dilated cardiomyopathy has a variety of causes such as: genetic factors, viral infections, alcohol abuse and myocardial infarction. Current classification of DCM excludes cases in which coronary artery disease, loading conditions, hypertension, valvular diseases and congenital heart diseases could have caused the observed phenotype (Elliott et al., 2008). The exact prevalence of idiopathic DCM varies between studies, from 1:2700 in the original epidemiological study (Codd et al., 1989) to 1:250 in a recent study (Hershberger et al., 2013). PPCM, as the name implies, develops during the last month of pregnancy, during delivery or within the

Abbreviations: ADMA, Asymmetric dimethylarginine; BR, Bromocriptine; $\mathrm{CD}$, Cathepsin D; DCM, Dilated cardiomyopathy; EF, Ejection Fraction; HF, Heart Failure; KO, Knock out; LMNA, Gene encoding the protein lamin-A/C; LV, Left ventricle; MnSOD, Manganese superoxide dismutase; NOS, Nitric oxide synthase; NOX, NADPH oxidase; PGC- $1 \alpha$, PPAR $\gamma$ coactivator- $1 \alpha$; PPCM, Peripartum cardiomyopathy; PRL, Prolactin; ROS, Reactive oxygen species; sFLT1, fms-like tyrosine kinase 1; STAT3, Signal transducer and activator of transcription 3; TTN, Gene encoding the protein titin; UPS, Ubiquitin proteasome system; VEGF, Vascular endothelial growth factor. first 5 months after delivery (Pearson et al., 2000). The prevalence of PPCM has been established to be 1:4000 to 1:1000 live births in the Western world (Elkayam, 2011; Kolte et al., 2014), although there is regional variation, e.g. the prevalence of PPCM in Haiti is 1:300 live births (Fett et al., 2005). In contrast to DCM, which typically presents later in life (Hershberger et al., 2013), PPCM often develops in young, previously healthy women and is a disease which progresses quickly to cardiac dysfunction and failure as is evident in the high mortality and transplantation rate (Van Hoeven et al., 1993; Hilfiker-Kleiner et al., 2007; Sliwa et al., 2010; Elkayam, 2011; Fett, 2014). As symptoms of PPCM such as dyspnea, fatigue and exercise incapacity are similar to normal pregnancy symptoms, the disease is often diagnosed late. If diagnosed and treated early, women suffering from PPCM may be stabilized upon treatment with $\beta$-blockers, ACE inhibitors and bromocriptine (BR) and reversal of the phenotype resulting in favorable outcome and recovery is not uncommon (Van Hoeven et al., 1993; Felker et al., 2000; Hilfiker-Kleiner et al., 2007; Sliwa et al., 2010; Elkayam, 2011; Ballo et al., 2012; Haghikia et al., 2013; Fett, 2014). This is contrary to what is often observed in DCM which is characterized by a late onset and slow disease progression that might be stabilized but unlikely to be reversed upon treatment (Van Hoeven et al., 1993; Fish, 2012; Hershberger et al., 2013).

\section{PHYSIOLOGICAL ADAPTATIONS DURING PREGNANCY}

The pathogenesis of PPCM is tightly bound to the cardiac changes that accompany normal pregnancies. It is therefore important to understand the physiological changes that occur during pregnancy. The increased hemodynamic load caused by the increased blood volume results in physiological hypertrophy, in which chamber dimensions increase, concomitant with a proportional increase in wall thickness to cope with the increased 
hemodynamic load and to facilitate increased cardiac output (Clapp and Capeless, 1997; Umar et al., 2012). This physiological hypertrophy is different from the pathological (eccentric) hypertrophy seen in both PPCM and DCM in which chamber dimensions increase while wall thickness does not increase proportionally in response to increased hemodynamic load (Gaasch and Zile, 2011). According to the law of Laplace this will lead to increased wall stress. Physiological pregnancy-related hypertrophy is reversible post-partum, usually within 1 year after delivery (Clapp and Capeless, 1997). Other differences between pathological and physiological hypertrophy are the absence of fibrosis and the absence of induction of the fetal gene program in physiological hypertrophy (McMullen and Jennings, 2007; Umar et al., 2012). In addition, angiogenic factors such as vascular endothelial growth factor A (VEGF A) are secreted during pregnancy which facilitates myocardial angiogenesis in order to increase capillary density proportionally to cardiomyocyte size. Both capillary density as well as VEGF levels return to pre-pregnancy levels post-partum (Umar et al., 2012; Chung and Leinwand, 2014). Pregnancy is often seen as a cardiac stress model upon which underlying cardiac dysfunction may reveal itself due to the increased hemodynamic load on the heart and hormonal changes during pregnancy (Chung and Leinwand, 2014). However, the increase in hemodynamic load starts early in pregnancy (Clapp and Capeless, 1997) while PPCM develops in the last month of pregnancy or even post-partum. It is therefore unlikely that the increased hemodynamic load is the sole cause of PPCM.

\section{GENETIC PREDISPOSITION AND TITIN ISOFORM SWITCHING}

As stated before, DCM can have a genetic cause, leading to a familial form of DCM. Many mutations have been linked to DCM. However, many of these genes are also implicated in hypertrophic and restrictive cardiomyopathies (Hershberger et al., 2013). A common gene which is mutated in $6 \%$ of DCM patients is the gene encoding for the protein lamin-A/C (LMNA) (Parks et al., 2008). LMNA mutations show a relatively high penetrance compared with mutations in other genes and patients carrying LMNA mutations often have conduction abnormalities (Parks et al., 2008; Hershberger et al., 2013). In addition, Herman et al. showed a high incidence of truncated variants in the gene encoding for the protein titin (TTN) in DCM patients (Herman et al., 2012). In PPCM, little is known about causative pathogenic mutations. However, the fact that multiple PPCM cases within one family have been reported (Van Spaendonck-Zwarts et al., 2014) suggests that gene mutations could play an important role. Furthermore, reports about DCM and PPCM cases within families and the identification of mutations (Morales et al., 2010; Van Spaendonck-Zwarts et al., 2010, 2014) strengthen the suggestion of a genetic cause and overlap in etiology of PPCM and DCM. A recent study showed a high incidence of TTN variants in PPCM patients, and this cohort was marked by slow recovery (Van Spaendonck-Zwarts et al., 2014). However, it has been proposed that TTN mutations are not always disease causing, but might act as disease modifiers as truncated TTN variants are present in 3\% of the general population (Herman et al., 2012). Knowledge about pathogenic effects of gene mutations would enable the identification of persons at risk for the development of DCM and PPCM and thereby facilitate early diagnosis and treatment.

The protein titin acts as a multifunctional spring that can exist as two distinct isoforms in the adult human heart; a compliant N2BA isoform and a stiff N2B isoform. A shift to more N2BA titin isoform and subsequent reduced passive stiffness was shown in DCM patients previously (Makarenko et al., 2004; Nagueh et al., 2004). Apart from isoform shift, alterations in titin posttranslational modifications such as phosphorylation are able to alter passive force development (Granzier and Labeit, 2002). Titin isoform has also been suggested to play a role in the ability of the heart to adapt contractility in response to stretch, known as the Frank-Starling mechanism (Fukuda et al., 2003). Unfortunately, limited data is available about the role of titin in PPCM, although increased compliant titin isoform and lowered passive tension has been reported in one PPCM patient with a TTN mutation (Van Spaendonck-Zwarts et al., 2014). Titin can also be modified under oxidizing conditions in which disulfide bridges can be formed in titin's N2B unique sequence possibly resulting in increased passive stiffness (Grützner et al., 2009). In addition, S-glutathionylation of cysteine residues in the Ig regions of titin under the influence of redox signaling has been suggested to lower passive stiffness (Alegre-Cebollada et al., 2014). As oxidative stress is present in both PPCM and DCM, as described later in this review, it is possible that this will also affect titin function although this has not been established in vivo yet.

\section{OXIDATIVE STRESS AND PROLACTIN: A DEADLY COMBINATION}

In both DCM and PPCM, oxidative stress is a key player in disease pathogenesis. However the exact consequences of reactive oxygen species (ROS) production differ notably between the two disease states as will be discussed below. In normal pregnancy, ROS production increases during the course of pregnancy and decreases post-partum to normal levels (Toescu et al., 2002). In an attempt to counterbalance the detrimental ROS production, total anti-oxidant capacity also increases during pregnancy and remains elevated post-partum (Toescu et al., 2002). In both PPCM animal models and human PPCM patients, oxidative stress levels are increased compared to healthy controls (HilfikerKleiner et al., 2007). An explanation for the increased oxidative stress in PPCM can be found in the PPCM mouse model with cardiomyocyte restricted deletion of Signal transducer and activator of transcription 3 (STAT3) (Hilfiker-Kleiner et al., 2007). This transcription factor regulates the expression of the superoxide scavenger Manganese superoxide dismutase (MnSOD) (Negoro et al., 2001). Accordingly, in the cardiac STAT3 KO mice PPCM is accompanied by a reduced expression of MnSOD and concomitant oxidative stress (Hilfiker-Kleiner et al., 2007). A crucial pathway in PPCM that is instigated by elevated oxidative stress is the cleavage of the hormone prolactin (PRL) by ROSactivated Cathepsin D (CD) (Hilfiker-Kleiner et al., 2007). Upon ROS activation CD cleaves full-length prolactin (PRL) of $23 \mathrm{kDa}$ into a smaller $16 \mathrm{kDa}$ form which has detrimental effects on cardiomyocyte metabolism and the microvasculature (HilfikerKleiner et al., 2007, 2012; Hilfiker-Kleiner and Sliwa, 2014). The 
idea that PRL plays a crucial role in PPCM is further strengthened by the fact that PRL levels rise at the end of pregnancy and remain high post-partum during breast feeding which coincides with the onset of PPCM (Grattan et al., 2008). Accordingly, injection of adenoviral vectors expressing $16 \mathrm{kDa}$ PRL in nonpregnant mice led to the development of cardiac dysfunction, dilation of the left ventricle (LV) and decreased myocardial capillary density (Hilfiker-Kleiner et al., 2007). As decreased levels of STAT3, high levels of oxidative stress, high CD activity and $16 \mathrm{kDa}$ PRL have also been observed in human PPCM patients (Hilfiker-Kleiner et al., 2007; Haghikia et al., 2013), it strengthens the suggestion that insufficient defense against oxidative stress and subsequent formation of $16 \mathrm{kDa}$ PRL plays an important role in PPCM pathogenesis. The compound BR is able to inhibit PRL release from the pituitary gland. Blockade of PRL by BR treatment in PPCM patients has been shown to improve cardiac function and increase survival, although only a limited number of studies containing small patient cohorts have been performed so far (Hilfiker-Kleiner et al., 2007; Sliwa et al., 2010; Ballo et al., 2012; Haghikia et al., 2013). Together these results indicate that cleavage of PRL under oxidative stress is key in the disease development in both human PPCM as well as the cardiac STAT-3 KO PPCM mouse-model (Hilfiker-Kleiner et al., 2007). However, not all patients treated with BR recover (Sliwa et al., 2010; Haghikia et al., 2013). Therefore, even though the PRL-mediated pathway is probably a major determinant in this disease, other pathways are likely to play a role. Since STAT3 signaling has many different functions, it is unlikely that the repressed MnSOD expression alone explains all observations in the STAT3 KO mouse model. Additionally, in heterozygous $\mathrm{MnSOD}^{+/-}$mice a reduction of MnSOD levels of 50\% causes cardiac alterations under basal conditions (Remmen et al., 2001) and induces cardiac remodeling after pregnancy (Hilfiker-Kleiner et al., 2007), but it does not lead to the dilated PPCM phenotype often seen in PPCM patients and the cardiac STAT3 KO mouse model. This implies that besides MnSOD additional factors are essential for the development of PPCM.

STATs are activated upon phosphorylation by Janus kinases (JAK) and are multifunctional transcription factors. In cardiomyocytes STAT3 is involved in survival, sarcomere integrity, cell growth and ROS production (Haghikia et al., 2014). In the vasculature STAT3 promotes vascularization through stimulation of VEGF signaling (Osugi et al., 2002). Clearly STAT3 affects multiple cardiac processes besides generation of the $16 \mathrm{kDa}$ PRL (Haghikia et al., 2014). In the cardiac STAT3 KO model, low STAT3 levels have been associated with the up-regulation of miR199 (Haghikia et al., 2011). MiR-199 down-regulates specific ubiquitin conjugating enzymes thereby affecting the ubiquitin proteasome system (UPS). In an in vitro model, this disturbance of the UPS resulted in decreased protein levels of myosin heavy chain and troponin $\mathrm{T}$, thereby causing disruption of sarcomere structure (Haghikia et al., 2011). Also in DCM patients, low STAT3 levels, increased miR-199 levels and decreased levels of ubiquitin conjugating enzymes have been observed (Haghikia et al., 2011). Low STAT3 levels have been observed in both PPCM (Hilfiker-Kleiner et al., 2007) and DCM patients (Podewski, 2003), indicating STAT3 may be part of the common pathway of both diseases. However, it should be noted that MnSOD protein levels and activity have been reported to be unchanged in DCM patients compared to non-failing controls in the myocardium (Dieterich et al., 2000) and a higher MnSOD activity was observed in serum (Wojciechowska et al., 2014). This indicates decreased STAT3 levels might have different downstream effects in DCM and PPCM and loss of MnSOD might form an etiology-specific (additional) source of oxidative stress in PPCM.

Oxidative stress is observed in many forms of heart failure (HF), including PPCM and DCM, and disturbs various pathways. However, the high levels of PRL during the final stages of pregnancy combined with high oxidative stress, likely make PPCM develop at such a high pace. As PRL levels are many fold lower in non-pregnant and non-nursing individuals (Grattan et al., 2008), it is unlikely that PRL also plays a role in DCM patients. Although oxidative stress has many detrimental effects in the failing heart it should be noted that ROS also fulfill a beneficial role in physiological circumstances. For example, NADPH oxidase (NOXs) produce ROS in a tightly regulated manner and play an important role in stretch-induced contraction by sensitization of the ryanodine receptor (RyR2) through oxidation (Prosser et al., 2011). However, in DCM NOX activity is up-regulated leading to increased ROS production (Maack et al., 2003). Amongst others oxidative stress inactivates important ion channels such as the L-type-calcium channel (LTCC) and the sarcoendoplasmic reticulum calcium transport ATPase (SERCA2a) and, in combination with disturbed RyR2 sensitization, may lead to $\mathrm{Ca}^{2+}$ depletion from the sarcoplasmic reticulum (Sag et al., 2013). Another general feature of HF is elevated cytosolic $\mathrm{Na}^{+}$levels (Despa, 2002) that result in decreased $\mathrm{Ca}^{2+}$ uptake and consequently reduced NADPH generation in mitochondria [28]. This disturbed balance of ions within cardiomyocytes causes cardiac dysfunction due to disturbed excitation-contraction coupling (Sag et al., 2013). In turn reduced mitochondrial NADPH availability leads to reduced generation of mitochondrial antioxidants and increased levels of ROS (Kohlhaas et al., 2010). In addition, ROS causes NADPH oxidation, thereby further reducing NADPH bioavailability for the production of ROS scavenger enzymes. In addition, ROS can reduce the bioavailability of tetrahydrobiopterin $\left(\mathrm{BH}_{4}\right)$ and thereby induce the uncoupling of nitric oxide synthase (NOS). As a result NOS no longer produces beneficial nitric oxide (NO) molecules, but itself becomes a producer of superoxide (Verhaar, 2004). In this way ROS production can be self-sustaining and cause additional ROS production (Seddon et al., 2007) thereby locking the heart in a vicious circle of oxidative stress (Kohlhaas et al., 2010). Similarly the NOS inhibitor asymmetric dimethylarginine (ADMA), a factor shown to be up-regulated in both DCM and PPCM and described later in this review, has been suggested to stimulate NOS-uncoupling mediated ROS production (Wilcox, 2012). However, it should be noted that ADMA levels at expected pathological concentrations only had modest effect on ROS production in this study (Druhan et al., 2008). The vicious circle of ROS production amplification and $\mathrm{Na}^{+} / \mathrm{Ca}^{2+}$ imbalance can be visualized in Figure 1. Apart from affecting cardiomyocyte ion balance, ROS has many other detrimental effects on cardiomyocytes such as nitration of creatine kinase, SERCA2a, voltage gated $\mathrm{K}^{+}$channels, desmin, myosin heavy 
chain and $\alpha$-actinin, induction of DNA damage, cell death and fibrosis (Pacher et al., 2007; Seddon et al., 2007). As fibrosis can increase myocardial stiffness and does not contribute to active force development, increased fibrosis can influence both systolic and diastolic function. Increased fibrosis is common in DCM patients (Assomull et al., 2006; Herpel et al., 2006) and the cardiac STAT3 KO mouse model of PPCM (Ricke-Hoch et al., 2014), however, there are limited and contradictory reports about fibrosis in PPCM patients (Kawano et al., 2008; Mouquet et al., 2008; Leurent et al., 2009; Ntusi and Chin, 2009).

Together, alterations induced by ROS can lead to cardiomyocyte dysfunction through impaired $\mathrm{Na}^{+}$and $\mathrm{Ca}^{2+}$ handling, impaired sarcomere integrity, cardiomyocyte death and fibrosis formation. Although $16 \mathrm{kDa}$ PRL is an important contributor to the pathophysiology of PPCM, it is likely that oxidative stressinduced cardiac pathology in PPCM also involve other ROS induced pathways.

\section{DISTURBED SARCOMERE INTEGRITY AND ANGIOGENIC IMBALANCE}

The previously described disturbance in the ubiquitin proteasome system (UPS) system also leads to increased levels of ADMA (Haghikia et al., 2011), a factor involved in reducing NO availability by competing with the NOS substrate L-arginine, and increasing ROS production by endothelial cells thereby contributing to the amplification of ROS production and cardiac dysfunction shown in Figure 1 (Druhan et al., 2008). Also in human PPCM (Haghikia et al., 2013) and DCM patients increased levels of ADMA have been found and in DCM decreased Larginine/ADMA ratio was shown to be a predictor of mortality (Anderssohn et al., 2012). The male mice of the cardiac STAT3 $\mathrm{KO}$ model showed age-related loss of capillary density and a DCM-like phenotype upon aging including fibrosis and impaired sarcomere organization (Hilfiker-Kleiner et al., 2004). Several studies indicate that damaged microvasculature or disturbance in

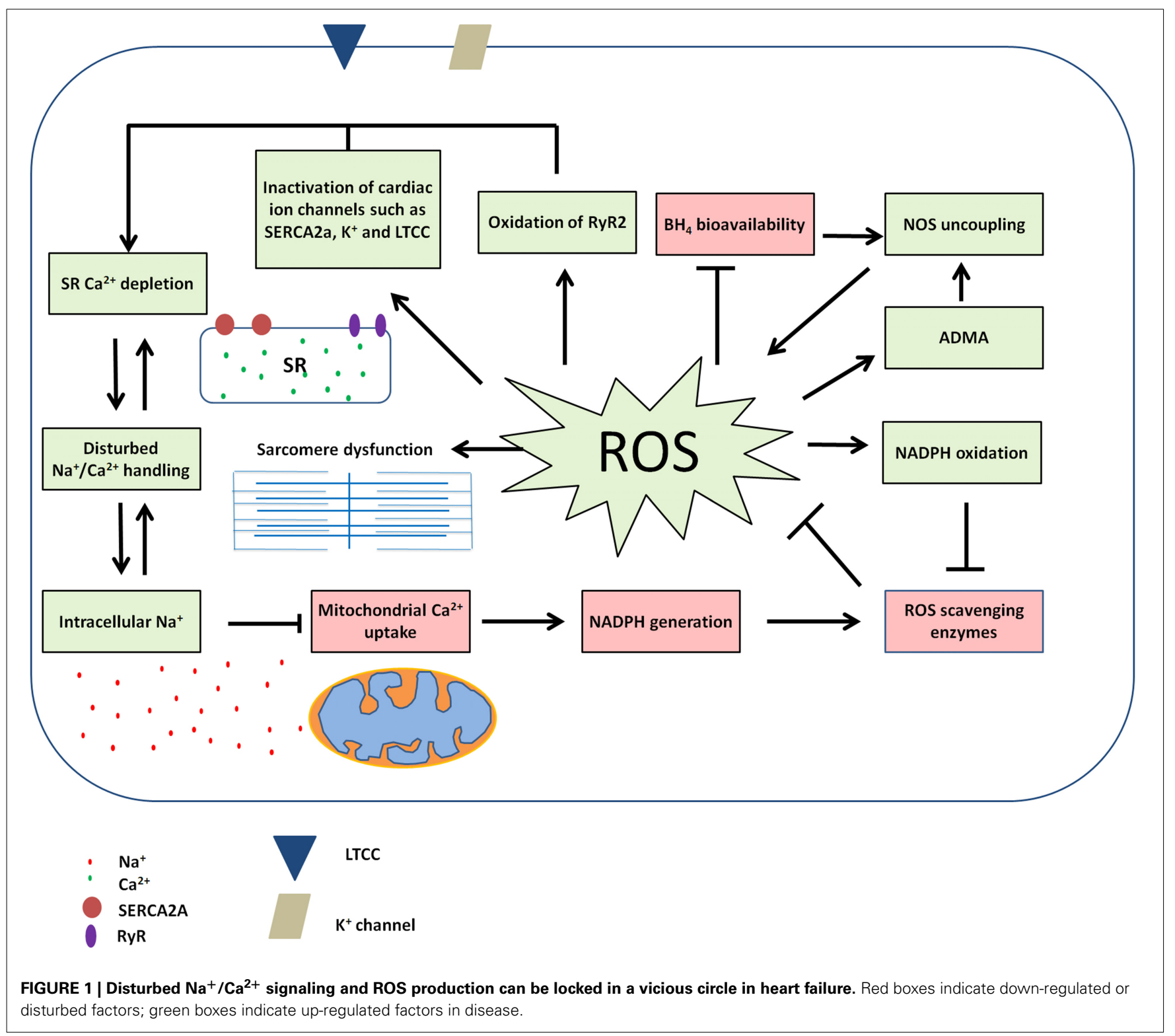


pro- and anti-angiogenic factors play a significant role in PPCM pathogenesis. A study by Patten et al. showed a high prevalence (33\%) of (pre)eclampsia in their PPCM cohort (Patten et al., 2012), which is believed to be a risk factor for PPCM. It has been shown that PPCM leads to capillary dissociation and endothelial cell apoptosis (Hilfiker-Kleiner et al., 2007). Patten et al. suggested that the anti-angiogenic state might worsen the severity of the disease (Patten et al., 2012). In late pregnancy, anti-angiogenic factors, such as soluble, fms-like tyrosine kinase 1 (sFLT1), are secreted and thereby inhibit the increased VEGF signaling during pregnancy (Chung and Leinwand, 2014). The increase in anti-angiogenic factors is significantly higher in patients with (pre)eclampsia which results in decreased VEGF levels (Levine et al., 2004) and also unusually high levels of sFLT1 have been observed in PPCM patients (Patten et al., 2012). This indicates the anti-angiogenic state is important in both (pre)eclampsia and PPCM pathogenesis and might explain the high prevalence of (pre)eclampsia among PPCM patients. A common finding in PPCM studies is a reduced capillary density in the postpartum phase (Hilfiker-Kleiner et al., 2007; Patten et al., 2012). Disturbed VEGF signaling and decreased capillary density have also been observed in DCM (Abraham et al., 2000; Karch et al., 2005; Lionetti et al., 2014). Another KO mouse model which lacks the cardiac PPAR $\gamma$ coactivator- $1 \alpha$ (PGC- $1 \alpha)$, also results in the development of PPCM (Patten et al., 2012). PGC-1 $\alpha$ promotes angiogenesis by stimulating VEGF signaling (Arany et al., 2008; Patten et al., 2012), (cardiac) mitochondrial biogenesis and metabolism in a tissue specific manner (Lehman et al., 2000; Arany et al., 2005). However, also in this model PPCM development occurs through the MnSOD, ROS and $16 \mathrm{kDa}$ PRL pathway as the phenotype could only be partly rescued by pro-angiogenic therapy in the form of VEGF A and treatment with both VEGF $A$ and BR was needed to obtain full recovery of LV function. Indeed, in PGC-1 $\alpha \mathrm{KO}$ mice MnSOD was reduced and ROS increased, which is not surprising as PGC- $1 \alpha$ is an inducer of MnSOD (Patten et al., 2012). Repressed levels of PGC-1 $\alpha$ have also been observed in PPCM patients (Patten et al., 2012). PGC$1 \alpha$ could also be implied in DCM as PGC- $1 \alpha \mathrm{KO}$ mice showed inability to increase heart rate in response to dobutamine, had markedly depressed levels of ATP in cardiac muscle and showed ventricle dilation and reduced fractional shortening upon aging (7-8 months), indicating PGC-1 $\alpha$ deficiency can induce a DCMlike phenotype (Arany et al., 2005). However, depressed levels of PGC-1 $\alpha$-regulated genes, but not expression levels of PGC$1 \alpha$ itself, have been reported in DCM (Sihag et al., 2009). The extend of depression of PGC- $1 \alpha$ regulated genes has been shown to be correlated to LV systolic function, which indicate mitochondrial function as regulated by PGC- $1 \alpha$ is impaired in $\mathrm{HF}$ (Sihag et al., 2009). The $16 \mathrm{kDa}$ PRL increases NFKB activity induced by miR-146 and thereby causes endothelial cell apoptosis (Tabruyn et al., 2003; Halkein et al., 2013). In addition, $16 \mathrm{kDa}$ PRL up-regulates exosome loading with miR-146 of endothelial cells. Uptake of these exosomes by cardiomyocytes causes miR146 induced impaired metabolic activity (Halkein et al., 2013). An interesting notion is that miR-146 has been shown to be upregulated in PPCM but not in DCM patients (Haghikia et al., 2013; Halkein et al., 2013). This indicates that although both patient groups suffer from dysfunctional microvasculature, the exact underlying pathogenesis might not be the same. Impaired microvasculature may result in insufficient oxygen and nutrient delivery to cardiomyocytes resulting in increased susceptibility to apoptosis and impaired cardiac function. It is clear that PPCM and DCM can result in similar clinical presentation even without the presence of PRL. The cleaved form of PRL is likely to cause an additional hit in PPCM quickly worsening disease progression and outcome. An overview of the changes in PPCM and DCM compared to healthy controls, grouped by downstream effect, can be seen in Table 1.

\section{PREGNANCY IN DCM PATIENTS AND SUBSEQUENT PREGNANCIES IN PPCM PATIENTS}

Given the similarities between PPCM and DCM, DCM patients presenting with the wish to conceive are a complicated patient group to counsel. Only a few studies have explored the disease alterations in DCM patients who become pregnant. The effects of pregnancy on cardiac function in DCM patients are contradictory as limited adverse events and recovery toward prepregnancy cardiac function (Bernstein and Magriples, 2001; Blatt et al., 2010), but also significant decreased cardiac event-free survival compared to non-pregnant DCM patients has been reported (Grewal et al., 2009). Adverse outcome in pregnant DCM patients was related to high NTproBNP levels, a known indicator for cardiac dysfunction, Blatt et al. (2010) and New York Heart Association functional classification class III or IV (Grewal et al., 2009). Most cardiac adverse events occurred at the beginning of the third trimester, which coincided with an increase in hemodynamic load on the heart (Grewal et al., 2009)

Table 1 | Altered parameters in PPCM and DCM compared to healthy controls.

\begin{tabular}{|c|c|c|c|}
\hline & Parameter & PPCM & DCM \\
\hline \multirow[t]{6}{*}{ Structure } & Chamber dilation & $\uparrow$ & $\uparrow$ \\
\hline & Sarcomere mutations & Possible & Possible \\
\hline & Sarcomere integrity & $\downarrow$ & $\downarrow$ \\
\hline & Fibrosis & $\begin{array}{l}\uparrow \text { in animals, } \\
\text { unknown in human }\end{array}$ & $\uparrow$ \\
\hline & Fetal gene program & Unknown & $\uparrow$ \\
\hline & Titin N2BA/N2B ratio & Unknown & $\uparrow$ \\
\hline \multirow{5}{*}{ Oxidative stress } & Oxidative stress & $\uparrow$ & $\uparrow$ \\
\hline & MnSOD & $\downarrow$ & $=$ \\
\hline & 16 kDa PRL & $\uparrow$ & Unlikely \\
\hline & PGC-1 $1 \alpha$ & $\downarrow$ & $\begin{array}{l}\downarrow \text { target gene } \\
\text { expression }\end{array}$ \\
\hline & STAT3 & $\downarrow$ & $\downarrow$ \\
\hline \multirow[t]{5}{*}{ Vasculature } & Capillary density & $\downarrow$ & $\downarrow$ \\
\hline & VEGF & $\downarrow$ & $\downarrow$ \\
\hline & miR-146 & $\uparrow$ & $=$ \\
\hline & miR-199 & $\uparrow$ & $\uparrow$ \\
\hline & ADMA & $\uparrow$ & $\uparrow$ \\
\hline
\end{tabular}

References for the shown parameters are indicated in the text. 
while PRL levels are still relatively low. Another case report that showed adverse events during pregnancy in a DCM patient also showed adverse events earlier in pregnancy than is observed in PPCM (Gevaert et al., 2014). Therefore, most adverse events seen in pregnant DCM patients are most likely due to the cardiac stress during pregnancy that is being placed on an already troubled heart. However, most adverse events were successfully treated and recovery to pre-pregnancy cardiac function was often established. A retrospective study by Bernstein et al. showed pregnancy is well-tolerated in stable DCM patients, while maternal outcome was significantly worse in PPCM patients (Bernstein and Magriples, 2001). Therefore, Bernstein et al. suggest the prognosis of PPCM patients should not be used when a stable DCM patient presents herself with the wish to conceive (Bernstein and Magriples, 2001). This strengthens the suggestion that although PPCM and DCM may share similar genetic predispositions and some common disease pathways, PPCM should not be considered as DCM occurring during or soon after pregnancy. However, it is also possible that pregnant DCM patients are protected from the fast deterioration of cardiac function seen in PPCM due to the fact that DCM patients are already treated for heart failure prior to and during pregnancy and are closely monitored. A small study by Hilfiker-Kleiner et al. showed BR treatment of PPCM patients in their subsequent pregnancy was able to improve survival and prevent relapse compared to PPCM patients without BR treatment (Hilfiker-Kleiner et al., 2007). Studies exploring subsequent pregnancies of PPCM patients revealed a HF relapse in 20-30\% and the ability to recover was related to cardiac function after PPCM index pregnancy and prior to the subsequent pregnancy (Elkayam et al., 2005; Fett et al., 2010). From these studies it can be concluded that stable DCM patients are unlikely to develop the fast PPCM disease progression and that the outcome of the DCM patient group is often more favorable than that of PPCM patients. The potential uneventful previous and subsequent pregnancies in PPCM patients indicate we do not know all triggers of PPCM since any predisposing mutation and PRL would also have been present in previous and subsequent event-free pregnancies. As PRL is present in both pregnant DCM patients and PPCM patients, we cannot simply state that PPCM is triggered by the presence of PRL in combination with a weakened heart.

\section{FUTURE PERSPECTIVES LARGE COHORT STUDIES PPCM}

At the moment there is still a lack of prospective studies exploring PPCM in larger cohorts. Most studies to date are case studies or small (often retrospective) cohort studies. A large, multicenter, randomized controlled clinical trial to evaluate BR treatment in 60 PPCM patients is ongoing, and the results should shed light on the effectiveness of BR treatment and outcome (US National Library of Medicine, 2012). In addition, the EURObservational Registry on PPCM has started to enroll patients that will be followed in a 1-year prospective study which will help to evaluate PPCM clinical presentations, risk factors, management and the effects on offspring worldwide (Sliwa et al., 2014).

\section{GENETIC PREDISPOSITIONS}

Information about disease initiation and predispositions such as pathogenic mutations is still limited in PPCM and DCM.
Therefore, more knowledge is needed on which mutations predispose people to DCM and PPCM and how these mutations exert their pathogenic effect in disease initiation and progression. Identification of these mutations would greatly enhance the ability to identify persons at risk to develop PPCM and DCM and by monitoring these patients early diagnosis and treatment could be facilitated thereby enhancing survival.

\section{CARDIAC REMODELING}

As cardiac deterioration, heart transplantations and death often occur within a few months after diagnosis in PPCM, it is possible that the cardiac remodeling to cope with the new cardiac state and demands in PPCM is different than in a more slow progressive disease as DCM. For example, in heart failure it has been shown that the fetal gene program is initiated resulting in cardiac remodeling in order to compensate for decreased cardiac function, although it contributes to cardiac dysfunction at a later stage (Thum et al., 2007). The limited time for adaptation of the heart might be detrimental in PPCM patients, as the lack of time to mount a compensatory response causes the need for cardiac transplantation, or lead to death soon after diagnosis. On the other hand, full recovery of PPCM patients may be explained by lack of permanent cardiac remodeling. However, not enough is known about remodeling of the PPCM heart and the recommendation to not use gadolinium in pregnant women has limited the use of magnetic resonance imaging (MRI) to assess fibrosis in PPCM patients. Therefore, other techniques to assess cardiac remodeling such as immunohistochemical staining of biopsies or explanted heart tissue should be explored in order to shed light on cardiac remodeling in PPCM.

\section{OXIDATIVE STRESS}

Based on observations in the STAT3 mouse model, mitigation of the intracellular defense mechanism against oxidative stress in human PPCM so far mainly focused on the reduction of MnSOD levels. Indeed reduction of MnSOD levels is an important contributor to elevated ROS levels in PPCM patients. However, to further unravel the patho-mechanism of oxidative stress in PPCM, future studies should involve the potential contribution of other enzymatic and non-enzymatic ROS scavengers as well as that of potential sources of ROS. Reduced MnSOD expression and PRL cleavage as a result of concomitant oxidative stress have been identified as important regulators of PPCM. Still the complete etiology of PPCM is far from completely understood.

\section{SUMMARY AND CONCLUSION}

PPCM and DCM likely share part of their pathogenesis such as predisposing mutations, increased oxidative stress, an impaired microvasculature and damaged sarcomere integrity. However, the exact underlying pathways might be differently altered in PPCM and DCM. While $16 \mathrm{kDa}$ PRL is likely to be a key player in PPCM it is unlikely to play a significant role in DCM. It is this $16 \mathrm{kDa}$ PRL that might explain the faster deterioration of cardiac function in PPCM by inducing an additional cascade of cardiovascular impairment. However, given the observation that pregnant DCM patients do better than PPCM patients, this difference in PRL levels between PPCM patients and nonpregnant DCM patients cannot explain all differences observed. 
The reports about uneventful subsequent pregnancies in PPCM patients indicate additional causative factors, such as insufficient defense mechanisms against oxidative stress or a vulnerable microvasculature. Therefore, despite their overlap in disease etiology and clinical presentation, differences in underlying pathways, disease progression and outcome argue for separation of the two disease states.

\section{ACKNOWLEDGMENTS}

We acknowledge support by the Netherlands organization for scientific research (NWO; VIDI grant 91711344) and the Rembrandt Institute.

\section{REFERENCES}

Abraham, D., Hofbauer, R., Schafer, R., Blumer, R., Paulus, P., Miksovsky, A., et al. (2000). Selective downregulation of VEGF-A165, VEGF-R1, and decreased capillary density in patients with dilative but not ischemic cardiomyopathy. Circ. Res. 87, 644-647. doi: 10.1161/01.RES.87.8.644

Alegre-Cebollada, J., Kosuri, P., Giganti, D., Eckels, E., Rivas-Pardo, J. A., Hamdani, N., et al. (2014). S-glutathionylation of cryptic cysteines enhances titin elasticity by blocking protein folding. Cell 156, 1235-1246. doi: 10.1016/j.cell.2014. 01.056

Anderssohn, M., Rosenberg, M., Schwedhelm, E., Zugck, C., Lutz, M., Lüneburg, N., et al. (2012). The L-Arginine-asymmetric dimethylarginine ratio is an independent predictor of mortality in dilated cardiomyopathy. J. Card. Fail. 18, 904-911. doi: 10.1016/j.cardfail.2012.10.011

Arany, Z., Foo, S.-Y., Ma, Y., Ruas, J. L., Bommi-Reddy, A., Girnun, G., et al. (2008). HIF-independent regulation of VEGF and angiogenesis by the transcriptional coactivator PGC-1alpha. Nature 451, 1008-1012. doi: 10.1038/nature06613

Arany, Z., He, H., Lin, J., Hoyer, K., Handschin, C., Toka, O., et al. (2005). Transcriptional coactivator PGC-1 alpha controls the energy state and contractile function of cardiac muscle. Cell Metab. 1, 259-271. doi: 10.1016/j.cmet.2005.03.002

Assomull, R. G., Prasad, S. K., Lyne, J., Smith, G., Burman, E. D., Khan, M., et al. (2006). Cardiovascular magnetic resonance, fibrosis, and prognosis in dilated cardiomyopathy. J. Am. Coll. Cardiol. 48, 1977-1985. doi: 10.1016/j.jacc.2006.07.049

Ballo, P., Betti, I., Mangialavori, G., Chiodi, L., Rapisardi, G., and Zuppiroli, A. (2012). Peripartum cardiomyopathy presenting with predominant left ventricular diastolic dysfunction: efficacy of bromocriptine. Case Rep. Med. 2012:476903. doi: 10.1155/2012/476903

Bernstein, P. S., and Magriples, U. (2001). Cardiomyopathy in pregnancy: a retrospective study. Am. J. Perinatol. 18, 163-168. doi: 10.1055/s-2001-14525

Blatt, A., Svirski, R., Morawsky, G., Uriel, N., Neeman, O., Sherman, D., et al. (2010). Short and long-term outcome of pregnant women with preexisting dilated cardiomypathy: an NTproBNP and echocardiography-guided study. Isr. Med. Assoc. J. 12, 613-616.

Chung, E., and Leinwand, L. A. (2014). Pregnancy as a cardiac stress model. Cardiovasc. Res. 101, 561-570. doi: 10.1093/cvr/cvu013

Clapp, J. F. I., and Capeless, E. (1997). Cardiovascular function before, during, and after the first and subsequent pregnancies. Am. J. Cardiol. 9149, 1469-1473. doi: 10.1016/S0002-9149(97)00738-8

Codd, M. B., Sugrue, D. D., Gersh, B. J., and Melton, L. J. (1989). Epidemiology of idiopathic dilated and hypertrophic cardiomyopathy. A populationbased study in Olmsted County, Minnesota. Circulation 80, 564-572. doi: 10.1161/01.CIR.80.3.564

Despa, S. (2002). Intracellular $\mathrm{Na}+$ concentration is elevated in heart failure but na/k pump function is unchanged. Circulation 105, 2543-2548. doi: 10.1161/01.CIR.0000016701.85760.97

Dieterich, S., Bieligk, U., Beulich, K., Hasenfuss, G., and Prestle, J. (2000). Gene expression of antioxidative enzymes in increased expression of catalase in the end-stage failing heart. Circulation 101, 33-39. doi: 10.1161/01.CIR.1 01.1 .33

Druhan, L. J., Forbes, S. P., Pope, A. J., Chen, C., Zweier, J. L., and Cardounel, A. J. (2008). Regulation of eNOS-derived superoxide by endogenous methylarginines †. 47, 7256-7263. doi: 10.1021/bi702377a
Elkayam, U. (2011). Clinical characteristics of peripartum cardiomyopathy in the United States: diagnosis, prognosis, and management. J. Am. Coll. Cardiol. 58, 659-670. doi: 10.1016/j.jacc.2011.03.047

Elkayam, U., Akhter, M. W., Singh, H., Khan, S., Bitar, F., Hameed, A., et al. (2005). Pregnancy-associated cardiomyopathy: clinical characteristics and a comparison between early and late presentation. Circulation 111, 2050-2055. doi: 10.1161/01.CIR.0000162478.36652.7E

Elliott, P., Andersson, B., Arbustini, E., Bilinska, Z., Cecchi, F., Charron, P., et al. (2008). Classification of the cardiomyopathies: a position statement from the European Society Of Cardiology Working Group on Myocardial and Pericardial Diseases. Eur. Heart J. 29, 270-276. doi: 10.1093/eurheartj/ehm342

Felker, G. M., Thompson, R. E., Hare, J. M., Hruban, R. H., Clemetson, D. E., Howard, D., et al. (2000). Underlying causes and long-term survival in patients with initially unexplained cardiomyopathy. N.Engl. J. Med. 342, 1077-1084. doi: 10.1056/NEJM200004133421502

Fett, J. D. (2014). Peripartum cardiomyopathy: a puzzle closer to solution. World J. Cardiol. 6, 87-99. doi: 10.4330/wjc.v6.i3.87

Fett, J. D., Christie, L. G., Carraway, R. D., and Murphy J. G. (2005). Five-year prospective study of the incidence and prognosis of peripartum cardiomyopathy at a single institution. Mayo Clin. Proc. 80, 1602-1606. doi: 10.4065/80.1 2.1602

Fett, J. D., Fristoe, K. L., and Welsh, S. N. (2010). Risk of heart failure relapse in subsequent pregnancy among peripartum cardiomyopathy mothers. Int. J. Gynaecol. Obstet. 109, 34-36. doi: 10.1016/j.ijgo.2009.10.011

Fish, W. (2012). Inherited cardiomyopathies. Br. J. Anaesth. 109, 643. author reply: 643-644. doi: 10.1093/bja/aes327

Fukuda, N., Wu, Y., Farman, G., Irving, T. C., and Granzier, H. (2003). Titin isoform variance and length dependence of activation in skinned bovine cardiac muscle. J. Physiol. 553, 147-154. doi: 10.1113/jphysiol.2003.049759

Gaasch, W. H., and Zile, M. R. (2011). Left ventricular structural remodeling in health and disease: with special emphasis on volume, mass, and geometry. J. Am. Coll. Cardiol. 58, 1733-1740. doi: 10.1016/j.jacc.2011.07.022

Gevaert, S., De Pauw, M., Tromp, F., Ascoop, A.-K., Roelens, K., and De Backer, J. (2014). Treatment of pre-existing cardiomyopathy during pregnancy. Acta Cardiol. 69, 193-196. doi: 10.2143/AC.69.2.3017302

Granzier, H., and Labeit, S. (2002). Cardiac titin: an adjustable multi-functional spring. J. Physiol. 541, 335-342. doi: 10.1113/jphysiol.2001.014381

Grattan, D. R., Steyn, F. J., Kokay, I. C., Anderson, G. M., and Bunn, S. J. (2008). Pregnancy-induced adaptation in the neuroendocrine control of prolactin secretion. J. Neuroendocrinol. 20, 497-507. doi: 10.1111/j.13652826.2008.01661.x

Grewal, J., Siu, S. C., Ross, H. J., Mason, J., Balint, O. H., Sermer, M., et al. (2009). Pregnancy outcomes in women with dilated cardiomyopathy. J. Am. Coll. Cardiol. 55, 45-52. doi: 10.1016/j.jacc.2009.08.036

Grützner, A., Garcia-Manyes, S., Kötter, S., Badilla, C. L., Fernandez, J. M., and Linke, W. A. (2009). Modulation of titin-based stiffness by disulfide bonding in the cardiac titin N2-B unique sequence. Biophys. J. 97, 825-834. doi: 10.1016/j.bpj.2009.05.037

Haghikia, A., Missol-Kolka, E., Tsikas, D., Venturini, L., Brundiers, S., Castoldi, M., et al. (2011). Signal transducer and activator of transcription 3-mediated regulation of miR-199a-5p links cardiomyocyte and endothelial cell function in the heart: a key role for ubiquitin-conjugating enzymes. Eur. Heart J. 32, 1287-1297. doi: 10.1093/eurheartj/ehq369

Haghikia, A., Podewski, E., Libhaber, E., Labidi, S., Fischer, D., Roentgen, P., et al. (2013). Phenotyping and outcome on contemporary management in a German cohort of patients with peripartum cardiomyopathy. Basic Res. Cardiol. 108, 366. doi: 10.1007/s00395-013-0366-9

Haghikia, A., Ricke-Hoch, M., Stapel, B., Gorst, I., and Hilfiker-Kleiner, D. (2014). STAT3, a key regulator of cell-to-cell communication in the heart. Cardiovasc. Res. 102, 281-289. doi: 10.1093/cvr/cvu034

Halkein, J., Tabruyn, S. P., Ricke-hoch, M., Haghikia, A., Nguyen, N., Scherr, M., et al. (2013). MicroRNA-146a is a therapeutic target and biomarker for peripartum cardiomyopathy. J. Clin. Invest. 123, 2143-2154. doi: 10.1172/JCI64365

Herman, D. S., Lam, L., Taylor, M. R. G., Wang, L., Teekakirikul, P., Christodoulou, D., et al. (2012). Truncations of titin causing dilated cardiomyopathy. N. Engl. J. Med. 366, 619-628. doi: 10.1056/NEJMoa1110186

Herpel, E., Pritsch, M., Koch, A., Dengler, T. J., Schirmacher, P., and Schnabel, P. A. (2006). Interstitial fibrosis in the heart: differences in extracellular matrix proteins and matrix metalloproteinases in end-stage dilated, ischaemic and 
valvular cardiomyopathy. Histopathology 48, 736-747. doi: 10.1111/j.13652559.2006.02398.x

Hershberger, R. E., Hedges, D. J., and Morales, A. (2013). Dilated cardiomyopathy: the complexity of a diverse genetic architecture. Nat. Rev. Cardiol. 10, 531-547. doi: 10.1038/nrcardio.2013.105

Hilfiker-Kleiner, D., Hilfiker, A., Fuchs, M., Kaminski, K., Schaefer, A., Schieffer, B., et al. (2004). Signal transducer and activator of transcription 3 is required for myocardial capillary growth, control of interstitial matrix deposition, and heart protection from ischemic injury. Circ. Res. 95, 187-195. doi: 10.1161/01.RES.0000134921.50377.61

Hilfiker-Kleiner, D., Kaminski, K., Podewski, E., Bonda, T., Schaefer, A., Sliwa, K., et al. (2007). A cathepsin D-cleaved $16 \mathrm{kDa}$ form of prolactin mediates postpartum cardiomyopathy. Cell 128, 589-600. doi: 10.1016/j.cell.2006.12.036

Hilfiker-Kleiner, D., and Sliwa, K. (2014). Pathophysiology and epidemiology of peripartum cardiomyopathy. Nat. Rev. Cardiol. 11, 364-370. doi: 10.1038/nrcardio. 2014.37

Hilfiker-Kleiner, D., Struman, I., Hoch, M., Podewski, E., and Sliwa, K. (2012). 16$\mathrm{kDa}$ prolactin and bromocriptine in postpartum cardiomyopathy. Curr. Heart Fail. Rep. 9, 174-182. doi: 10.1007/s11897-012-0095-7

Karch, R., Neumann, F., Ullrich, R., Neumüller, J., Podesser, B. K., Neumann, M., et al. (2005). The spatial pattern of coronary capillaries in patients with dilated, ischemic, or inflammatory cardiomyopathy. Cardiovasc. Pathol. 14, 135-144. doi: 10.1016/j.carpath.2005.03.003

Kawano, H., Tsuneto, A., Koide, Y., Tasaki, H., Sueyoshi, E., Sakamoto, I., et al. (2008). Magnetic resonance imaging in a patient with peripartum cardiomyopathy. Intern. Med. 47, 97-102. doi: 10.2169/internalmedicine.47.0316

Kohlhaas, M., Liu, T., Knopp, A., Zeller, T., Ong, M. F., Böhm, M., et al. (2010). Elevated cytosolic $\mathrm{Na}+$ increases mitochondrial formation of reactive oxygen species in failing cardiac myocytes. Circulation 121, 1606-1613. doi: 10.1161/CIRCULATIONAHA.109.914911

Kolte, D., Khera, S., Aronow, W. S., Palaniswamy, C., Mujib, M., Ahn, C., et al. (2014). Temporal trends in incidence and outcomes of peripartum cardiomyopathy in the United States: a nationwide population-based study. J. Am. Heart Assoc. 3:e001056. doi: 10.1161/JAHA.114.001056

Lehman, J. J., Barger, P. M., Kovacs, A., Saffitz, J. E., Medeiros, D. M., and Kelly, D. P. (2000). Peroxisome proliferator-activated receptor gamma coactivator-1 promotes cardiac mitochondrial biogenesis. J. Clin. Invest. 106, 847-856. doi: 10.1172/JCI10268

Leurent, G., Baruteau, A. E., Larralde, A., Ollivier, R., Schleich, J. M., Boulmier, D., et al. (2009). Contribution of cardiac MRI in the comprehension of peripartum cardiomyopathy pathogenesis. Int. J. Cardiol. 132, e91-e93. doi: 10.1016/j.ijcard.2007.12.012

Levine, R. J., Maynard, S. E., Qian, C., Lim, K.-H., England, L. J., Yu, K. F., et al. (2004). Circulating angiogenic factors and the risk of preeclampsia. N. Engl. J. Med. 350, 672-683. doi: 10.1056/NEJMoa031884

Lionetti, V., Matteucci, M., Ribezzo, M., Di Silvestre, D., Brambilla, F., Agostini, S., et al. (2014). Regional mapping of myocardial hibernation phenotype in idiopathic end-stage dilated cardiomyopathy. J. Cell. Mol. Med. 18, 396-414. doi: $10.1111 /$ jcmm. 12198

Maack, C., Kartes, T., Kilter, H., Schäfers, H.-J., Nickenig, G., Böhm, M., et al. (2003). Oxygen free radical release in human failing myocardium is associated with increased activity of racl-GTPase and represents a target for statin treatment. Circulation 108, 1567-1574. doi: 10.1161/01.CIR.0000091084.46 500.BB

Makarenko, I., Opitz, C. A., Leake, M. C., Neagoe, C., Kulke, M., Gwathmey, J. K., et al. (2004). Passive stiffness changes caused by upregulation of compliant titin isoforms in human dilated cardiomyopathy hearts. Circ. Res. 95, 708-716. doi: 10.1161/01.RES.0000143901.37063.2f

McMullen, J. R., and Jennings, G. L. (2007). Differences between pathological and physiological cardiac hypertrophy: novel therapeutic strategies to treat heart failure. Clin. Exp. Pharmacol. Physiol. 34, 255-262. doi: 10.1111/j.14401681.2007.04585.x

Morales, A., Painter, T., Li, R., Siegfried, J. D., Li, D., Norton, N., et al. (2010). Rare variant mutations in pregnancy-associated or peripartum cardiomyopathy. Circulation 121, 2176-2182. doi: 10.1161/CIRCULATIONAHA.109.931220

Mouquet, F., Lions, C., de Groote, P., Bouabdallaoui, N., Willoteaux, S., Dagorn, J., et al. (2008). Characterisation of peripartum cardiomyopathy by cardiac magnetic resonance imaging. Eur. Radiol. 18, 2765-2769. doi: 10.1007/s00330008-1067-x
Nagueh, S. F., Shah, G., Wu, Y., Torre-Amione, G., King, N. M. P., Lahmers, S., et al. (2004). Altered titin expression, myocardial stiffness, and left ventricular function in patients with dilated cardiomyopathy. Circulation 110, 155-162. doi: 10.1161/01.CIR.0000135591.37759.AF

Negoro, S., Kunisada, K., Fujio, Y., Funamoto, M., Darville, M. I., Eizirik, D. L., et al. (2001). Activation of signal transducer and activator of transcription 3 protects cardiomyocytes from hypoxia/reoxygenation-induced oxidative stress through the upregulation of manganese superoxide dismutase. Circulation 104, 979-981. doi: 10.1161/hc3401.095947

Ntusi, N. B., and Chin, A. (2009). Characterisation of peripartum cardiomyopathy by cardiac magnetic resonance imaging. Eur. Radiol. 19, 1324-1325. author reply: 1326-1327. doi: 10.1007/s00330-008-1244-y

Osugi, T., Oshima, Y., Fujio, Y., Funamoto, M., Yamashita, A., Negoro, S., et al. (2002). Cardiac-specific activation of signal transducer and activator of transcription 3 promotes vascular formation in the heart. J. Biol. Chem. 277, 6676-6681. doi: 10.1074/jbc.M108246200

Pacher, P., Beckman, J. S., and Liaudet, L. (2007). Nitric oxide and peroxynitrite in health and disease. Physiol. Rev. 87, 315-424. doi: 10.1152/physrev.00029.2006

Parks, S. B., Kushner, J. D., Nauman, D., Burgess, D., Ludwigsen, S., Peterson, A., et al. (2008). Lamin A/C mutation analysis in a cohort of 324 unrelated patients with idiopathic or familial dilated cardiomyopathy. Am. Heart J. 156, 161-169. doi: 10.1016/j.ahj.2008.01.026

Patten, I. S., Rana, S., Shahul, S., Rowe, G. C., Jang, C., Liu, L., et al. (2012). Cardiac angiogenic imbalance leads to peripartum cardiomyopathy. Nature 485, 333-338. doi: 10.1038/nature11040

Pearson, G. D., Veille, J.-C., Rahimtoola, S., Hsia, J., Oakley, C. M., Hosenpud, J. D., et al. (2000). Peripartum cardiomyopathy:national heart, lung and blood institute and office of rare diseases (national institutes of health) workshop recommendations and review. JAMA 283, 1183-1188. doi: 10.1001/jama.283.9.1183

Podewski, E. K. (2003). Alterations in Janus Kinase (JAK)-signal transducers and activators of transcription (STAT) signaling in patients with end-stage dilated cardiomyopathy. Circulation 107, 798-802. doi: 10.1161/01.CIR.0000057545.82749.FF

Prosser, B. L., Ward, C. W., and Lederer, W. J. (2011). X-ROS signaling: rapid mechano-chemo transduction in heart. Science 333, 1440-1445. doi: 10.1126/science. 1202768

Remmen, H. V., Williams, M. D., Guo, Z., Estlack, L., Yang, H., Carlson, E. J., et al. (2001). Knockout mice heterozygous for Sod2 show alterations in cardiac mitochondrial function and apoptosis. Am. J. Physiol. Heart Circ. Physiol. 281, H1422-H1432.

Ricke-Hoch, M., Bultmann, I., Stapel, B., Condorelli, G., Rinas, U., Sliwa, K., et al. (2014). Opposing roles of Akt and STAT3 in the protection of the maternal heart from peripartum stress. Cardiovasc. Res. 101, 587-596. doi: 10.1093/cvr/cvu010

Sag, C. M., Wagner, S., and Maier, L. S. (2013). Role of oxidants on calcium and sodium movement in healthy and diseased cardiac myocytes. Free Radic. Biol. Med. 63, 338-349. doi: 10.1016/j.freeradbiomed.2013.05.035

Seddon, M., Looi, Y. H., and Shah, A. M. (2007). Oxidative stress and redox signalling in cardiac hypertrophy and heart failure. Heart 93, 903-907. doi: 10.1136/hrt.2005.068270

Sihag, S., Cresci, S., Li, A. Y., Sucharov, C. C., and Lehman, J. J. (2009). PGC-1alpha and ERRalpha target gene downregulation is a signature of the failing human heart. J. Mol. Cell. Cardiol. 46, 201-212. doi: 10.1016/j.yjmcc.2008.10.025

Sliwa, K., Blauwet, L., Tibazarwa, K., Libhaber, E., Smedema, J.-P., Becker, A., et al. (2010). Evaluation of bromocriptine in the treatment of acute severe peripartum cardiomyopathy: a proof-of-concept pilot study. Circulation 121, 1465-1473. doi: 10.1161/CIRCULATIONAHA.109.901496

Sliwa, K., Hilfiker-Kleiner, D., Mebazaa, A., Petrie, M. C., Maggioni, A. P., RegitzZagrosek, V., et al. (2014). EURObservational research programme: a worldwide registry on peripartum cardiomyopathy (PPCM) in conjunction with the Heart Failure Association of the European Society of Cardiology Working Group on PPCM. Eur. J. Heart Fail. 16, 583-591. doi: 10.1002/ejhf.68

Tabruyn, S. P., Sorlet, C. M., Rentier-Delrue, F., Bours, V., Weiner, R. I., Martial, J., et al. (2003). The antiangiogenic factor $16 \mathrm{~K}$ human prolactin induces caspasedependent apoptosis by a mechanism that requires activation of nuclear factorkappaB. Mol. Endocrinol. 17, 1815-1823. doi: 10.1210/me.2003-0132

Thum, T., Galuppo, P., Wolf, C., Fiedler, J., Kneitz, S., van Laake, L. W., et al. (2007). MicroRNAs in the human heart: a clue to fetal gene reprogramming in heart failure. Circulation 116, 258-267. doi: 10.1161/CIRCULATIONAHA.107.687947 
Toescu, V., Nuttall, S. L., Martin, U., Kendall, M. J., and Dunne, F. (2002). Oxidative stress and normal pregnancy. Clin. Endocrinol. (Oxf.) 57, 609-613. doi: 10.1046/j.1365-2265.2002.01638.x

Umar, S., Nadadur, R., Iorga, A., Amjedi, M., Matori, H., and Eghbali, M. (2012). Cardiac structural and hemodynamic changes associated with physiological heart hypertrophy of pregnancy are reversed postpartum. J. Appl. Physiol. 113, 1253-1259. doi: 10.1152/japplphysiol.00549.2012

US National Library of Medicine. (2012). ClinicalTrials.Gov. Available online at: http://clinicaltrials.gov/ct2/show/NCT00998556

Van Hoeven, K. H., Kitsis, R. N., Katz, S. D., and Factor, S. M. (1993). Peripartum versus idiopathic dilated cardiomyopathy in young women-a comparison of clinical, pathologic and prognostic features. Int. J. Cardiol. 40, 57-65. doi: 10.1016/0167-5273(93)90231-5

Van Spaendonck-Zwarts, K. Y., Posafalvi, A., van den Berg, M. P., Hilfiker-Kleiner, D., Bollen, I. A. E., Sliwa, K., et al. (2014). Titin gene mutations are common in families with both peripartum cardiomyopathy and dilated cardiomyopathy. Eur. Heart J. 35, 2165-2173. doi: 10.1093/eurheartj/ehu050

Van Spaendonck-Zwarts, K. Y., van Tintelen, J. P., van Veldhuisen, D. J., van der Werf, R., Jongbloed, J. D. H., Paulus, W. J., et al. (2010). Peripartum cardiomyopathy as a part of familial dilated cardiomyopathy. Circulation 121, 2169-2175. doi: 10.1161/CIRCULATIONAHA.109.929646

Verhaar, M. C. (2004). Free radical production by dysfunctional eNOS. Heart 90, 494-495. doi: 10.1136/hrt.2003.029405

Wilcox, C. S. (2012). Asymmetric dimethylarginine and reactive oxygen species: unwelcome twin visitors to the cardiovascular and kidney disease tables. Hypertension 59, 375-381. doi: 10.1161/HYPERTENSIONAHA.111.1 87310

Wojciechowska, C., Romuk, E., Tomasik, A., Nowalany-kozielska, E., Birkner, E., and Jache, W. (2014). Oxidative stress markers and C-reactive protein are related to severity of heart failure in patients with dilated Cardiomyopathy. 2014:147040 doi: 10.1155/2014/147040

Conflict of Interest Statement: The authors declare that the research was conducted in the absence of any commercial or financial relationships that could be construed as a potential conflict of interest.

Received: 05 December 2014; accepted: 29 December 2014; published online: 15 January 2015.

Citation: Bollen IAE, Van Deel ED, Kuster DWD and Van Der Velden J (2015) Peripartum cardiomyopathy and dilated cardiomyopathy: different at heart. Front. Physiol. 5:531. doi: 10.3389/fphys.2014.00531

This article was submitted to Striated Muscle Physiology, a section of the journal Frontiers in Physiology.

Copyright (C) 2015 Bollen, Van Deel, Kuster and Van Der Velden. This is an openaccess article distributed under the terms of the Creative Commons Attribution License (CC BY). The use, distribution or reproduction in other forums is permitted, provided the original author(s) or licensor are credited and that the original publication in this journal is cited, in accordance with accepted academic practice. No use, distribution or reproduction is permitted which does not comply with these terms. 